\title{
Heterotaxy syndrome and biliodigestive anastomosis with migration of biliar prosthesis
}

\begin{abstract}
Background: Endoscopic stent insertion is a primary technique for the decompression of obstructed biliopancreatic duct system. Distal migration of biliary stents is considered a rare complication. When this happens, prostheses normally pass spontaneously through the gastrointestinal tract or remain in the intestine without symptoms, but sometimes the migration of the stent can lead to impaction and cause problems. A biliodigestive anastomosis is a surgical connection between the common bile duct and the digestive tract to prevent interference of bile flow. This surgery may be performed for several different reasons, including management of the injury of the biliary tract. Roux-en-Y hepaticojejunostomy is currently considered as the definitive treatment for iatrogenic bile duct injuries and the principal representative of biliary diversion procedures.
\end{abstract}

Heterotaxy is a rare syndrome characterized by an abnormal arrangement of the internal organs in the chest and abdomen. Despite its variable presentation, heterotaxy syndrome can be classified into heterotaxy with polysplenia or left isomerism, and heterotaxy with asplenia or right isomerism.

Case presentation: We present the case of a 42-year-old male with heterotaxy with polysplenia who underwent laparoscopic cholecystectomy for cholelithiasis. The reconstruction of the bile duct was performed as a result of the complete section of the common hepatic duct during surgery. The next six years, he presented with recurrent episodes of cholangitis that required dilation of the stenosis and the placement of biliary stents through ERCP. However, the patient required a Roux-en-Y biliodigestive anastomosis during which distal migration of the biliary stent occurred, The prosthesis remained in the duodenum for several months without showing clinical symptoms, until it was removed by endoscopy using a polypectomy handle.

Conclusion: This is a unique case in the literature for heterotaxy and the patient's surgical history, in which distal migration of the biliary stent occurs during surgical manipulation in the biliodigestive shunt. The jejunojejunal anastomosis is an area where the stent can easily be stopped.

Keywords: Biliary tract, stents, foreign-body migration, anastomosis Roux-en-Y, heterotaxy syndrome, polysplenia syndrome
Volume II Issue I - 2020

\author{
José Maximiliano Garófano-Jerez, ${ }^{1,2,3}$ Elena \\ Benedicto-Hernández, ${ }^{4}$ Juan de Dios López- \\ González Gila, ${ }^{5}$ Antonio Paulino Garófano- \\ Jerez, ${ }^{6}$ Juan de Dios López-González \\ Garrido ${ }^{1,2}$ \\ 'Department of Radiology and Physical Medicine, University of \\ Granada, Spain \\ ${ }^{2}$ Faculty of Medicine, University of Granada, Spain \\ ${ }^{3}$ Radiagnostic Service, University Hospital San Cecilio de \\ Granada, Spain \\ ${ }^{4}$ Radiodiagnostic Service, University Regional Hospital of Málaga, \\ Spain \\ ${ }^{5}$ Nephrology Service, University Hospital Virgen de las Nieves \\ de Granada, Spain \\ ${ }^{6}$ Radiodiagnostic Service, University Hospital of Jaén, Spain
}

Correspondence: Juan de Dios López-González Garrido, University Professor. Department of Radiology and Physical Medicine of the Faculty of Medicine.Avda. De la Investigación I I, CP. I80I6 Granada, Spain, Tel +34 958244246, Email jdlopezg@ugr.es

Received: December 04, 2019 | Published: January 03, 2019
Abbreviations: CT, computed tomography; MDCT, Multidetector Computed Tomography; ERCP, rndoscopic retrograde cholangiopancreatography

\section{Introduction}

\section{Heterotaxy syndrome with polysplenia}

Heterotaxy syndrome, or situs ambiguus, refers to the abnormal arrangement of organs and vessels in the thorax and abdomen relative to midline, ${ }^{1-4}$ and is usually associated with complex heart malformations. Malformations in other organs can also be found. The diagnosis of the heterotaxy syndrome can be complex due to its variable presentation $^{5}$ and although there are characteristic findings, none of them is pathognomonic. Despite the fact that a specific pattern cannot be identified in most cases, there is a tendency to bilateral visceroatrial symmetry, ${ }^{6}$ which allows differentiation in two categories: heterotaxy with left isomerism (polysplenia) and heterotaxy with right isomerism (asplenia).
Situs ambiguus with polysplenia, or left isomerism, is the most common form of heterotaxy and has female predominance. ${ }^{7}$ Left isomerism is generally characterized by centrally located abdominal organs and presence of multiple spleens; however, one single lobulated spleen or even a normal spleen is seen in some patients. The spleen or spleens are always on the same side as the stomach, typically along its greater curvature.

The most consistent anatomic finding is an interrupted suprarenal $\mathrm{IVC}^{7}$ with azygous or hemiazygous continuation. Cardiac anomalies in polysplenic patients are less severe and less common (50-90\%) than in patients with situs ambiguus with asplenia. In patients with heterotaxy syndrome with polysplenia the liver and gallblader are susally located next to midline (Figures 3-5). ${ }^{2}$ Anomalies of the biliary tract generally include biliary atresia in neonates and gallblader hypoplasia or agenesis in infancy. As for the pancreas, the most common anomaly found in polysplenic patients is truncated pancreas (Figure 5). ${ }^{2}$ Intestinal malrotation is also common ( $70 \%$ of patients approximately) (Figures 4-6). ${ }^{7}$ 


\section{Roux-en-Y hepaticojejunostomy}

A hepaticojejunostomy is the surgical creation of a communication between the hepatic duct and the jejunum. The major indications for hepaticojejunostomy are benign or iatrogenic strictures, and injuries to the biliary system.

Roux-en-Y hepaticojejunostomy is sometimes performed for biliary reconstruction (Figure 1). For anastomosis to either the common bile duct or the confluence of the hepatic ducts, it is necessary to fashion a Roux-en-Y jejunal limb. ${ }^{8}$ This limb is identified and transected with a stapling device $30-35 \mathrm{~cm}$ from the ligament of Treitz. To achieve a stable anastomosis, mucosal contact must exist between the bowel and the biliary tree.

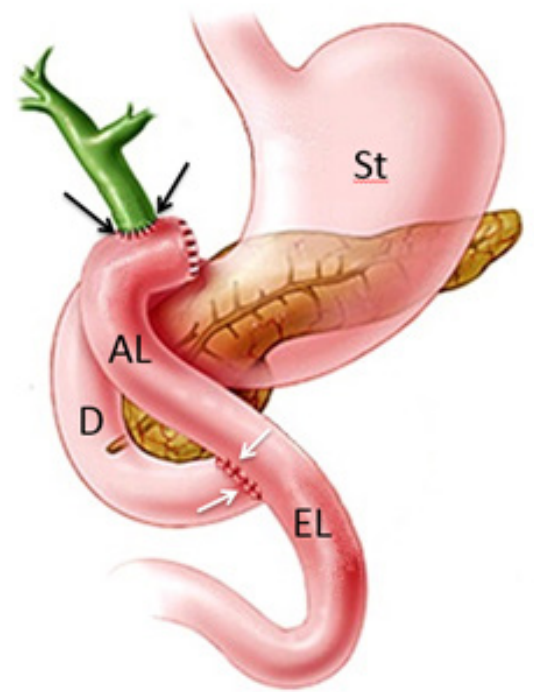

Figure I Schematic representation of Roux-en-Y hepaticojejunostomy. Stomach (St), duodenum (D), Roux limb or jejunal afferent loop (AL), jejunal eferent loop (EL), hepaticojejunal anastomosis (black arrows), jejunojenunal anastomosis (white arrows).

The two upper limbs of the Y represent: the proximal segment of stomach and the proximal small bowel it joins with; and the blind end that is surgically divided off. And the lower part of the $\mathrm{Y}$ is formed by the distal small bowel beyond the anastomosis. The alimentary limb remains the primary recipient of food after the surgery, while the hepatobiliary or afferent limb that anastomoses with the biliary system serves as the recipient for biliary secretions, which then travel through the excluded small bowel to the distal anastomosis at the mid jejunum (efferent limb) to aid digestion. There are two anastomoses: a proximal hepaticojejunal anastomosis and a distal jejunojejunal anastomosis.

\section{Biliary stents}

Endoscopic insertion of biliary endoprostheses is a common and useful technique for the decompression of obstructed pancreatic and biliary ducts. This effective procedure is conducted with low rates of morbidity and complications, such us cholangitis, cholecystitis, duodenal perforation, hemorrhages, pancreatitis, stent breakage, proximal and distal migration, and stent occlusion. ${ }^{9-11}$

Stents may unfold incorrectly or may shift after a variable time interval after placement. Migration seems to occur more frequently with coated stents, probably due to their insufficient anchorage.
Although generally spontaneous, stent migration can sometimes be caused by surgical (as in the presented case), endoscopic or percutaneous maneuvers.

Stent migration is a rare complication, occurring in $5-10 \%$ of cases of malignancies, and the incidence may increase in benign processes due to inflammation. ${ }^{9,12,13}$ Distally migrated stents usually pass spontaneously through the gastrointestinal tract ( $86 \%$ of cases) or remain in the intestine without any symptoms. ${ }^{10,14-16}$ The duodenum is the most common site of stent migration due to its proximity to the biliary tree and its retroperitoneal fixation. ${ }^{10,11}$ Papillary stenosis, omission of sphincterotomy, use of plastic or coated metallic stents, and stents in benign lesions have been associated with distal migration. ${ }^{11,15}$

The position of a misplaced or migrated stent is often overlooked, especially when the patient is asymptomatic. However, rapid recognition of these stents is important because repositioning or removal of the device, percutaneously or endoscopically, can be performed to prevent the development of complications such as penetration, perforation and obstruction. ${ }^{9,10,14,15}$ The presence of patient abdominal comorbidities, for example, adhesions, diverticular disease and/or hernias, is associated to a higher risk of complications. ${ }^{10,11,15,17}$

Patients with biliary endoprostheses should be followed up clinically and with imaging, endoscopy or x-ray tests. ${ }^{3}$ In patients with biliary prostheses and abdominal pain or sepsis, differential diagnosis with perforation should be considered and abdominal x-rays performed.10 In these cases, the most common site of perforation is the duodenum and perforations are usually retroperitoneal. ${ }^{9,18}$

Although biliary prostheses can be evaluated by conventional radiographic studies (Figure 2), computed tomography (CT) has several distinct advantages over them in the visualization of the migrated stents and in the diagnosis of associated complications. Multidetector Computed Tomography (MDCT) provides high-resolution images not only of the endoprosthesis, but also of the wall of the biliary or enteric segments where the stent and surrounding fat planes, organs and other abdominal structures are located. While endoscopy and conventional radiography show only intraluminal changes, CT can be used to visualize extraluminal signs of complications such as free peritoneal air, retroperitoneal gas, or liquid collections. The major limitations of MDCT are the radiation dose, which is cumulative, and the high cost, especially if this imaging technique is performed only for the evaluation of a permanent stent. ${ }^{19}$

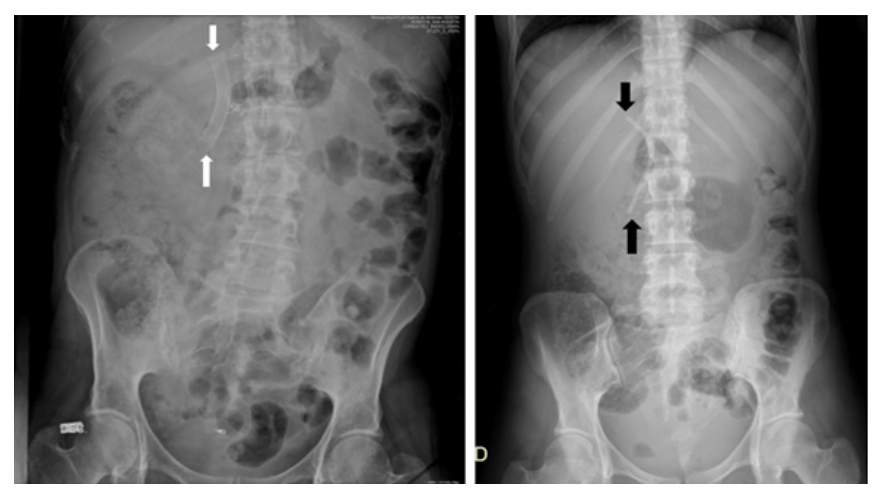

Figure 2 Simple X-rays of abdomen of other patients, with well-positioned metallic (white arrows) and plastic (black arrows) biliary stents. 


\section{Management of distal migration}

A. Prosthesis migration into the duodenum

i. Management depends on the location of the prosthesis, whether it is in the peritoneal or retroperitoneal space, and the patient clinical presentation. ${ }^{20}$

ii. In general, in uncontained lateral duodenal wall perforations, the prosthesis must be removed immediately, and major surgery is required. However, small, contained retroperitoneal perforations can be conservatively managed with antibiotics and clinical and radiographic surveillance.

B. Prosthesis migration into the colon

i. In the absence of impaction or perforation, two approaches are possible: ${ }^{17}$

a. Expectant treatment, since most foreign bodies pass through the intestine without incident. This requires thorough and prolonged follow-up with repeated abdominal x-rays.

b. Immediate endoscopic extraction in patients with diverticulosis or inguinal hernia, as they have a higher risk of perforation.

ii. Colonic migrations that cause perforation used to be treated surgically, but now surgery is performed in the presence of peritonitis or fistulas. ${ }^{16}$ In cases of limited perforation and absence of peritonitis, endoscopic extraction has been proposed as the standard treatment. ${ }^{17}$

In order to avoid complications, it is generally recommended that the migrated prosthesis should be removed immediately, either by endoscopic, percutaneous or mixed technique. ${ }^{21}$ The surgical approach is no longer recommended and it is reserved for situations when previous techniques have failed. ${ }^{11,15,21}$

\section{Presentation of case}

A 42-year-old man with heterotaxy syndrome with polysplenia, underwent laparoscopic cholecystectomy for cholelithiasis, after which, reconstruction of the bile duct is performed secondary to a complete section of the common hepatic duct. Further CT and magnetic resonance cholangiography images confirm the diagnosis of situs ambiguus with polysplenia showing the following distribution and morphology of the thoracoabdominal organs: ${ }^{1}$

a. Polysplenia with splenuli on the left side (Figures 3-4).

b. iver in midline, with a prominent left hepatic lobe (Figures 3-5).

c. Hepatic venous drainage directly to the atrium by means of a common trunk due to the absence of the hepatic segment of the ICV (Figure 3).

d. Stomach in normal position on the left (Figures 3-5).

e. Truncated or incomplete pancreas (only the region of the head is visualized) (Figure 4).

f. Intestinal malrotation: the duodenum does not pass through the aortomesenteric angle, the Treitz angle is not visualized, the colon is in the right hemiabdomen, and the small intestine in the left hemiabdomen (Figures 4-5).

g. Difficult identification of the infrarenal ICV, with multiple serpiginous vessels in retroperitoneum, presumably venous, next to the aorta (Figure 4).

h. Right aortic artery (Figures 3-4).

i. Abdominal blood flow returns via the azygos venous system (a large azygos vein is located to the left of the thoracic aorta artery) (Figure 3).

j. Dextrocardia with cardiac apex to the right (Figures 3 and 5).
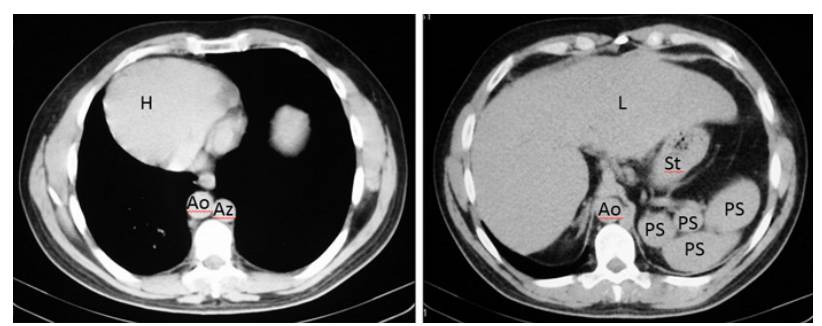

Figure 3 Axial CT images show dextrocardia and cardiac apex on the right $(\mathrm{H})$, the aorta on the right (Ao), azygos vein on the left $(A z)$, liver is midline (L) but slightly on the right and polysplenia (PS) on the left relative to greater curvature of the stomach (St). IVC cannot be identified.
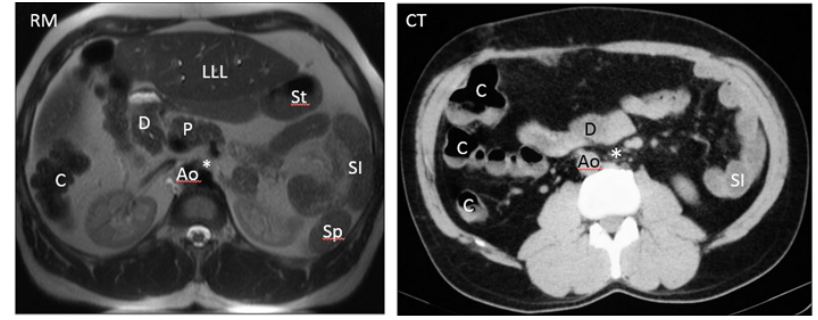

Figure 4 Axial MR and CT images show a prominent left hepatic lobe (LLL), colon on the right (C), small intestine on the left (SI), truncated pancreas $(\mathrm{P})$, left-sided stomach $(\mathrm{St})$, one splenule on the left $(\mathrm{Sp})$, Aorta on the right (Ao) and intestinal malrotation because the duodenum (D) does not pass between the aorta and the superior mesenteric artery (asterisk). IVC cannot be identified.

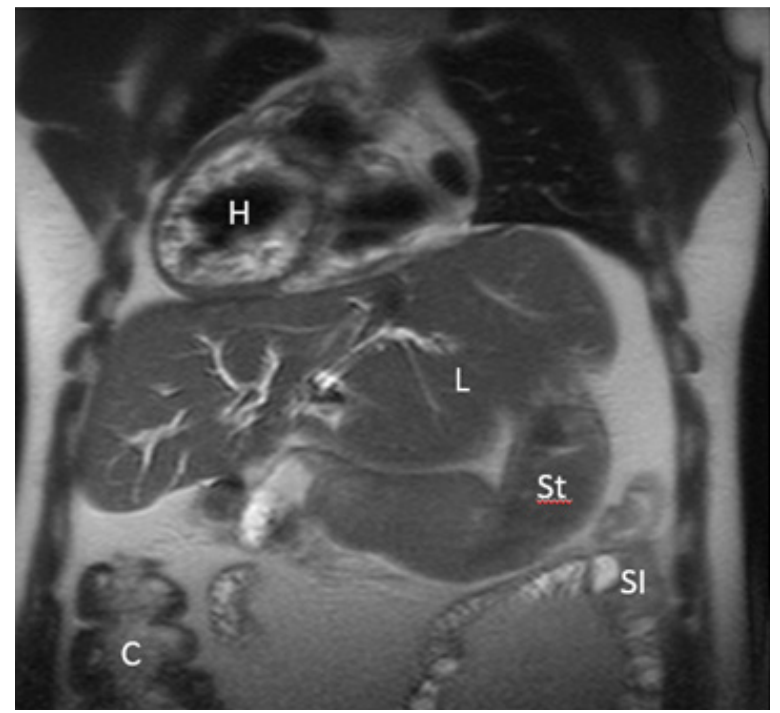

Figure 5 Coronal MR image shows dextrocardia and cardiac apex on the right $(H)$, midline liver $(L)$ with a prominent left lobe, colon on the right $(C)$, small intestine on the left (SI). Stomach (St). 
During the six years following laparoscopic cholecystectomy, the patient undergoes frequent medical examinations due to multiple episodes of acute cholangitis that require endoscopic dilatation of the stenosis and placement of biliary stents through endoscopic retrograde cholangiopancreatographies (ERCP) until, finally, the patient had a biliodigestive (Roux-en-Y) anastomosis. During surgery, the stent is not removed as it is not visualized and, based on the suspicion of a possible distal migration, a radiograph of the abdomen is requested, in which the prosthesis seems to be found in the hepatic angle of the colon (Figure 6). Over five months the patient was asymptomatic, and the stent, presumably in transit, was controlled through radiographs but it does not seem to change its location. In view of the above, it is decided to perform a computerized tomography to accurately locate the prosthesis (Figures 7-8), it was shown that it was located in the duodenum with the distal end in the region of the jejunal anatomy, since it had not managed to circumvent the angulation that occurs in the efferent jejunal loop just after the anastomosis.

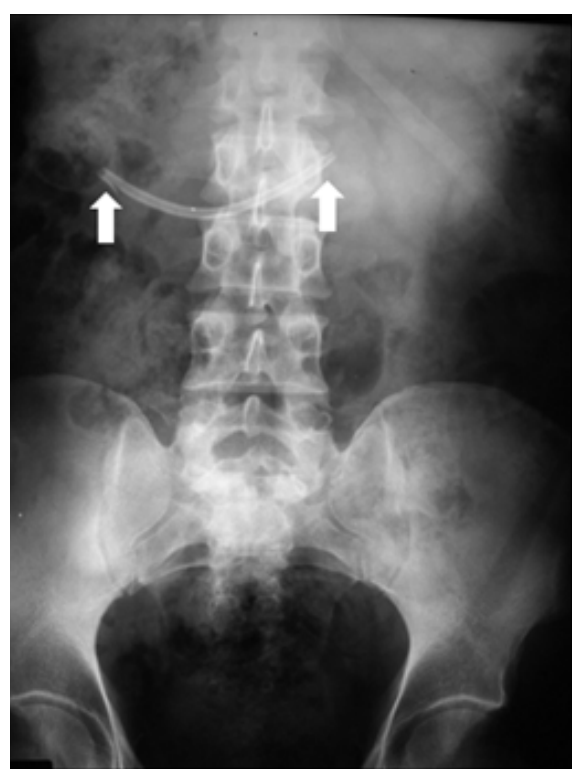

Figure 6 Abdominal radiography shows the displaced stent (arrows).

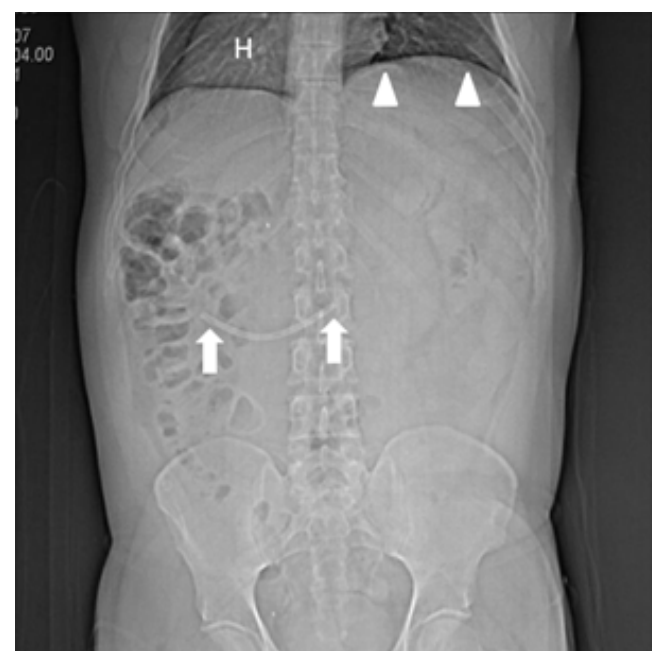

Figure $7 \mathrm{CT}$ projection radiograph (topogram) shows dextrocardia with cardiac apex on the right $(\mathrm{H})$, left diaphragm in a superior position (arrowheads) and the displaced stent (arrows) in a similar location than that seen in the previous figure.
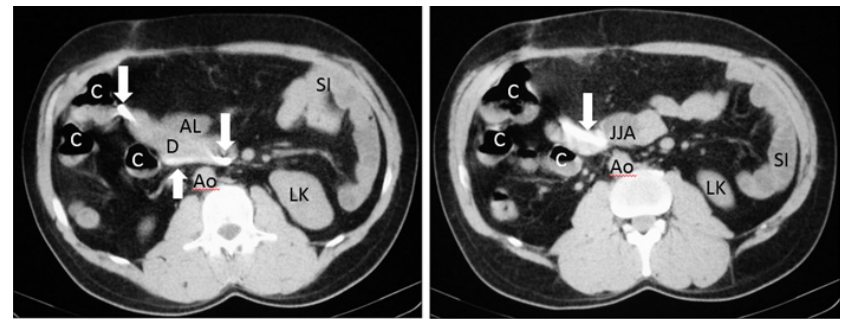

Figure 8 Axial CT images showing the high density migrated stent (arrows), located in the duodenum (D) with end in the jejunonojejunal anastomosis $(J J A)$. Jejunal afferent loop (AL), left kidney (LK), colon (C), small intestine (SI), aorta (Ao).

As mentioned above, in order to avoid complications, stent extraction is recommended and, due to the exceptional situation of our patient, that same day the prosthesis was endoscopically removed from the duodenum with a polypectomy handle.

\section{Discussion}

It should be noted that in heterotaxy syndrome with polysplenia, the liver and gallbladder are usually located near the midline.,22 Abnormalities of the biliary tract generally involve biliary atresia in neonates and gallbladder hypoplasia or agenesis in childhood. ${ }^{5}$ Abnormalities of the biliary system are rarely described in adults because only $5-10 \%$ of patients live into adolescence.

In the presented case, we believe that there must have been an abnormal location of the gallbladder and adjacent structures making laparoscopic cholecystectomy difficult, which resulted in a complete section of the common hepatic duct. In addition, the subsequent complications (bile duct stenosis, recurrent cholangitis), which required ERCP stent placement, resulted in the distal migration of the stent towards the small intestine during the Roux-en-Y hepaticojejunostomy performed to definitively solve the described complications.

Stent migration is considered a rare event, occurring in 5-10\% of malignant processes, and with higher incidence in benign processes due to inflammation, ${ }^{9,12,13}$ as in our patient. In addition, migration has been reported to be more frequent with plastic prostheses, ${ }^{11}$ used in benign stenosis, as in this patient (Figure 7). Distally migrated stents normally pass through the gastrointestinal tract ( $86 \%$ of cases). ${ }^{10,14,15,16}$ For this reason, initial management for the patient was expectant, with thorough and prolonged follow-up by abdominal x-rays ${ }^{17}$ (Figure 6). A lower percentage of prostheses remain in the intestine without symptoms ${ }^{10,14,15,16}$ as in the presented case. Thus, after a 5-month follow-up with abdominal x-rays, where no significant changes were observed in the location of the prosthesis, it was decided to perform an abdominal CT scan, both to locate the stent and to provide the endoscopist with detailed information of the abnormal post-surgical anatomy of the patient's upper gastrointestinal tract. ${ }^{23}$ Lastly, the prosthesis was localized in the duodenum with its distal end in the area of jejunojejunal anastomosis (Figure 8). We understand that at the beginning of the surgical Roux-en-Y hepaticojejunostomy there was a migration of the prosthesis, which remained in the duodenum until the end of the procedure. Subsequently, due to the angle in the segment of the anastomosis, the prosthesis failed to progress through this area remaining in the same location during the five months of follow-up (Figure 9). 


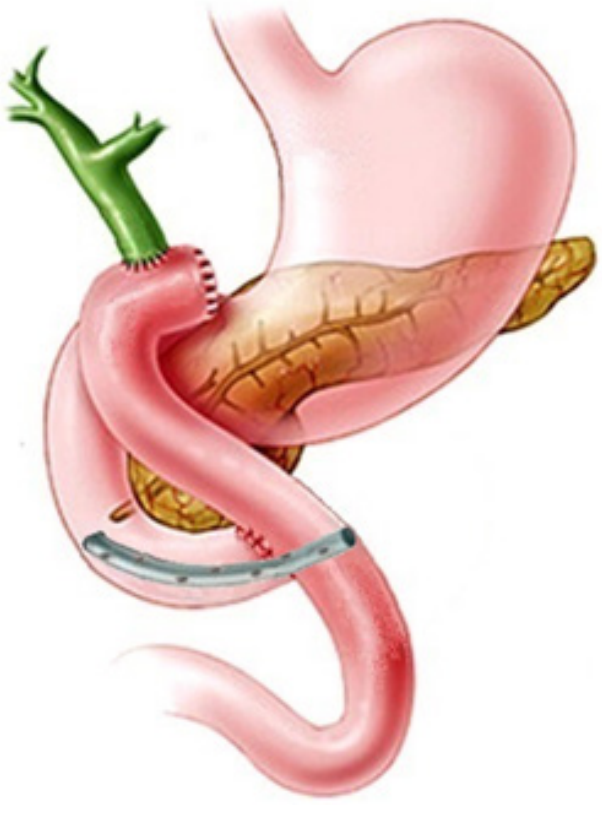

Figure 9 Schematic representation of Roux-en-Y hepaticojejunostomy with the displaced stent located in the duodenum and jejunojejunal anastomosi.

In the review of the literature we found reports of metal stents used to treat stenosis in the gastric outlet after gastric by-passes (Rouxen-Y), which have migrated and impacted the jejunal anastomosis, ${ }^{24}$ although they also refer to metal stents used in similar situations, which have managed to pass through the jejunal anastomosis, being evacuated later through the rectum. As for the extraction of the stent, and despite the patient being asymptomatic at all times, we think the follow-up (five months) was excessive, and that the extraction should have been performed after the first radiographic control in which the absence of change in the position of the prosthesis was noted, since the risk of possible impaction and intestinal perforation would make this advisable.

\section{Conclusion}

This is a unique case, since we have not been able to find in the literature any report of patients with heterotaxy suffering distal migration of a biliary stent during the performance of a Roux-en-Y hepaticojejunostomy, and that the stent remains in place in the area of the jejunojejunal anastomosis. We believe that the anatomical changes in the liver and gallbladder may have favoured section of the common hepatic duct during laparoscopic cholecystectomy, but we do not think that heterotaxy is related to the subsequent migration of the biliary stent.

This distal migration of the stent was caused by surgical manipulation during the Roux-en-Y hepaticojejunostomy. The jejunojejunal anastomosis, although it can be crossed by a stent, is an area where the stent can be stopped more easily than in other areas of the intestine.

\section{Acknowledgments}

To Dr. Martín Ruiz, since his concern about the patient's health has allowed this case was solved.

\section{Conflicts of interest}

Authors declare that there is no conflict of interest.

\section{Funding}

None.

\section{References}

1. Garófano-Jerez JM, Benedicto-Hernández E, López-González Gila $\mathrm{JD}$, et al. Imaging evaluation of the heterotaxy syndrome. Gastroenterol Hepatol Open Access. 2019;10(2):75-80.

2. Corral GG, Labra WA, Schiappacasse FG. Abdominal manifestations of abnormalities of Situs Ambiguous in adults: Regarding four cases. Rev Chil Radiol. 2013;19(1):38-43.

3. Kim SJ. Heterotaxy syndrome. Korean Circ J. 2011;41(5):227-232

4. Cupers S, Van Linthout C, Desprechins B, et al. Heterotaxy syndrome with intestinal malrotation, polysplenia and azygos continuity. Clin Pract. 2018;8(1):1004

5. Prieto Martínez C, Vila Costas JJ, García Sanchotena JL, et al. Heterotaxy syndrome (polysplenia) in an adult. Radiology. 2007;49(3):211-214.

6. Civetta JD, Cayré RO, Obregón R, et al. Visceroatrial situs: its importance in the segmental diagnosis of congenital heart disease. Argentina: National University of the Northeast; 2006.

7. Mujo T, Finnegan T, Joshi J, et al. Situs ambiguous, levocardia, right sided stomach, obstructing duodenal web, and intestinal nonrotation: a case report. J Radiol Case Rep. 2015;9(2):16-23.

8. Felder SI, Menon VG, Nissen NN, et al. Hepaticojejunostomy using short-limb Roux-en-Y reconstruction. JAMA Surg. 2013;148(3):253257; discussion 257-258.

9. Siaperas P, Ioannidis A, Skarpas A, et al. A rare cause for Hartmann's procedure due to biliary stent migration: A case report. Int J Surg Case Rep. 2017;31:83-85.

10. Mady RF, Niaz OS, Assal MM. Migrated biliary stent causing perforation of sigmoid colon and pelvic abscess. BMJ Case Rep. 2015;2015:10.1136/bcr-2014-206805.

11. Malgras B, Pierret C, Tourtier J, et al. Double sigmoid colon perforation due to migration of a biliary stent. J Visc Surg. 2011;148(5):e397-e399.

12. Kawaguchi Y, Ogawa M, Kawashima Y, et al. Risk factors for proximal migration of biliary tube stents. World J Gastroenterol. 2014;20(5):13181324.

13. Güitrón A, Adalid R, Barinagarrementeria R, et al. Proximal migration of biliary prostheses. Endoscopic extraction techniques. Rev Gastroenterol Mex. 2001;66(1):22-26.

14. Namdar T, Raffel AM, Topp SA, et al. Complications and treatment of migrated biliary endoprostheses: a review of the literature. World $J$ Gastroenterol. 2007;13(40):5397-5399.

15. Garg K, Zagzag J, Khaykis I, et al. Surgical relief of small bowel obstruction by migrated biliary stent: extraction without enterotomy. JSLS. 2011;15(2):232-235.

16. Jafferbhoy SF, Scriven P, Bannister J, et al. Endoscopic management of migrated biliary stent causing sigmoid perforation. BMJ Case Rep. 2011;2011:10.1136/bcr.04.2011.4078.

17. Virgilio E, Pascarella G, Scandavini CM, et al. Colonic perforations caused by migrated plastic biliary stents. Korean J Radiol. 2015;16 (2):444-445. 
18. Smart P, Keenan D, Campbell W, et al. Duodenal perforation secondary to migration of endobiliary prosthesis. BMJ Case Rep. 2012;2012:10.1136/ bcr-2012-006711.

19. Catalano O, De Bellis M, Sandomenico F, et al. Complications of Biliary and Gastrointestinal Stents: MDCT of the Cancer Patient. AJR Am J Roentgenol. 2012;198:W1-W10.

20. Kundu R, Pleskow D. Biliary and Pancreatic Stents: Complications and Management. Techn Gastrointest Endosc. 2007;9(2):125-134.

21. Lichtenstein DR. Post-Surgical Anatomy and ERCP. Techn Gastrointest Endosc. 2007;9(2):114-124.
22. Fulcher AS, Turner MA. Abdominal manifestations of situs anomalies in adults. Radiographics. 2002;22(6):1439-1456.

23. Güngör G, Okur N. A Fatal Complication: Intestinal Perforation Secondary to Migration of a Biliary Stent. Pol J Radiol. 2016;81:170 172 .

24. Marcotte É, Corneau É, Meziat-Burdin A, et al. Early migration of fully covered double-layered metallic stents for post-gastric bypass anastomotic strictures. Intern J Case Report. 2012;3(7):283-286. 